\title{
Methylation of the promoter of human leukocyte antigen class I in human esophageal squamous cell carcinoma and its histopathological characteristics
}

\author{
Sun Qifeng, MD, Cong Bo, MD, Jiang Xingtao, MD, Peng Chuanliang, MD, and Zhao Xiaogang, MD
}

Objective: The downregulation of human leukocyte antigen class I (HLA-I) has been proposed to contribute to
the immune evasion of cancer cells in some cancers. Meanwhile, transcriptional silencing by means of promoter
methylation is now believed to be an important mechanism of carcinogenesis. The aim of this study was (1) to
examine the expression of HLA-I antigen and the antigen-processing machinery components in patients with
esophageal squamous cell carcinoma lesions and (2) to detect the methylation pattern of the HLA-I gene in pa-
tients with esophageal squamous cell carcinoma and assess its association with histopathological characteristics.

Methods: A total of 87 formalin-fixed and paraffin-embedded esophageal squamous cell carcinoma lesions were collected. HLA-I and antigen-processing machinery component expression was investigated by means of immunohistochemistry with anti-HLA-I monoclonal antibody, and methylation changes in the promoter region of HLA-1 genes were determined by using methylation-specific polymerase chain reaction.

Results: HLA-I, transporter associated with antigen processing 1, and low molecular weight protein were lost or downregulated in $67 \%, 29.8 \%$, and $47.0 \%$ of the esophageal squamous cell carcinoma lesions, respectively. The positive rates of gene promoter hypermethylation of HLA-I was 70.1\% (61/87) in tumor tissues compared with $3.6 \%$ in normal tissue $(P<.01)$. Also, the higher methylation rates and the HLA-I expression were significantly associated with tumor grade, including lymph node metastasis $(P<.05)$.

Conclusions: HLA-I promoter hypermethylation was associated with loss of HLA-I antigen, which frequently occurred in primary tumors, especially in metastatic lymph node lesions, and was associated with patients' prognoses. (J Thorac Cardiovasc Surg 2011;141:808-14)

Esophageal squamous cell carcinoma (ESCC) is a very aggressive disease with a poor prognosis. Despite aggressive treatment modalities, such as surgical tumor resection with extensive lymphadenectomy and chemoradiation therapy, only $20 \%$ to $36 \%$ of patients survive at 5 years. ${ }^{1}$ In recent years, immunotherapy, such as the use of antitumor $\mathrm{T}$ cells or antibodies induced by cancer vaccination, has become an attractive option for the revival of the role of immune surveillance in the control of tumor growth. ${ }^{2}$ It is well known that human leukocyte antigen class I (HLA-I) molecules are critical for the presentation of antigen peptides, including those derived from tumor cells, to cytotoxic T lymphocytes, and the downregulation of HLA-I on the tumor allows it to evade cytotoxic T lymphocyte-mediated antitumor immunity, leading to variant cancer cells that arise from the parent tumor during tumor progression at both primary and metastatic sites. ${ }^{3}$ Loss of HLA-I antigen expression can occur at the genetic, transcrip-

From Thoracic Surgery, Second Hospital of Shandong University, Jinan, China Disclosures: Authors have nothing to disclose with regard to commercial support.

Received for publication Jan 31, 2010; revisions received April 26, 2010; accepted for publication April 30, 2010.

Address for reprints: Zhao Xiaogang, MD, Thoracic Surgery, Second Hospital of Shandong University, 247 Beiyuan St, Jinan, Shandong 250033, China (E-mail: menglin2002@163.com).

0022-5223/\$36.00

Copyright $₫ 2011$ Published by Elsevier Inc. on behalf of The American Association for Thoracic Surgery

doi:10.1016/j.jtcvs.2010.04.031 tional, and posttranscriptional levels. DNA methylation, especially $5^{\prime} \mathrm{CpG}$ methylation, is an important mechanism in silencing the expression of genes. ${ }^{4,5}$ DNA methylation can directly interfere with the basal transcriptional machinery by altering the DNA secondary structure, especially the major groove conformation. DNA methylation can also induce chromosomal remodeling through histone deacetylation, resulting in transcriptional repression. These events suggest that DNA hypermethylation should be an important role in the downregulation of HLA-I genes in patients with ESCC. To our knowledge, there have been few reports describing HLA-I expression in patients with ESCC and few describing its mechanism, especially DNA methylation. Thus in this study we investigated HLA-I expression in primary and metastatic lesions in patients with ESCC using immunohistochemistry. Second, we investigated the methylation changes in the promoter region of HLA-I by using methylation-specific polymerase chain reaction (PCR). Furthermore, we evaluated the correlation between methylation of HLA-I and the clinicopathological status or prognosis of patients with ESCC.

\section{MATERIALS AND METHODS}

\section{ESCC Specimen Preparation and DNA Extraction}

Eighty-seven primary ESCC specimens containing neighboring epithelial tissue were collected at the Second Hospital of Shandong University in Jinan (Table 1). The samples were frozen in liquid nitrogen within 1 hour after surgical resection and were stored in liquid nitrogen on dry ice 


\section{Abbreviations and Acronyms \\ APM $=$ antigen-processing machinery \\ ESCC $=$ esophageal squamous cell carcinoma \\ HLA-I = human leukocyte antigen class I \\ LMP2 = low molecular weight protein 2 \\ $\mathrm{PCR}=$ polymerase chain reaction \\ TAP1 $=$ transporter associated with antigen processing 1}

at $-80^{\circ} \mathrm{C}$ until use. For each specimen, 2 pieces of tissue, one from the tumor mass, were dissected and embedded with tissue freezing medium (optimal cutting temperature compound). Ten-micrometer-thick serial sections were cryosected and used for DNA extraction. The presence of tumor or normal tissue in the samples was confirmed histopathologically on slides stained with hematoxylin and eosin. Cancerous lesions were microdissected with a laser-capture microdissection system for the genetic assay. About 500 cells were dissected for each lesion. DNA was extracted with a Qiagen Tissue Kit, according to the manufacturer's procedures. The DNA was stored in aliquots at $-20^{\circ} \mathrm{C}$ until use. None of the patients received radiotherapy, chemotherapy, or other medical interventions before the study. This study was approved by the Ethics Committee of the Shandong University, and written informed consent was obtained from all subjects.

\section{Immunohistochemical Staining}

Immunohistochemical staining was performed with the PV6000 IHC Kit. Briefly, sections were cut at $5 \mathrm{~mm}$ from the tissue microarray blocks. After being dewaxed in xylene and rehydrated in alcohol and distilled water, antigen was retrieved by means of microwave oven heating (10 minutes) at middle power in $0.01 \mathrm{~mol} / \mathrm{L}$ sodium citrate buffer $(\mathrm{pH} 6.0)$. The sections were then incubated with $3 \%$ hydrogen peroxide in absolute methanol at room temperature for 10 minutes to block endogenous peroxidase activity. After 3 rinses (each for 5 minutes) in phosphate-buffered saline, the sections were incubated sequentially with murine anti-human HLA-I antibody (1:100; Proteintech Group), a rabbit anti-human transporter associated with antigen processing 1 (TAP1) antibody (1:100, Proteintech Group), and a murine anti-human low molecular weight protein 2 (LMP2) antibody (1:100; Proteintech Group) diluted in phosphate-buffered saline overnight at $48^{\circ} \mathrm{C}$ with $150 \mathrm{~mL}$ of polymerized horseradish peroxidase-anti-mouse/rabbit IgG for 30 minutes at room temperature. The reaction products were visualized with diaminobenzidine (Peking Zhongshan), and slides were counterstained with hematoxylin, dehydrated, and evaluated under light microscope. Negative controls were done by omitting primary antibodies. For the evaluation of HLA-I expression, 2 independent observers assessed HLA-I positivity, without previous knowledge of clinicopathological data.

A consensus number was reached for each tumor sample between the 2 investigators. Normal lymphocytes and vessel endothelia were used in each specimen as internal controls. Tumor specimens were scored as positive, heterogeneous, or negative when the percentage of stained tumor cells in an entire lesion was greater than $75 \%, 25 \%$ to $75 \%$, and less than $25 \%$, respectively, according to the criteria established by the HLA and cancer component of the 12th International Histocompatibility Workshop. ${ }^{6}$

\section{Methylation-Specific PCR and Sequencing}

The tumor and normal DNA were modified by means of a bisulfite reaction with the procedure developed by Herman. ${ }^{7}$ For microdissected DNA, $1 \mu \mathrm{g}$ of salmon sperm DNA was added as a carrier, followed by denaturation with $\mathrm{NaOH}$ (final concentration, $0.2 \mathrm{~mol} / \mathrm{L}$ ). The DNA, in a volume of 50 $\mu \mathrm{L}$, was incubated with $275 \mu \mathrm{L}$ of modification buffer ( $10 \mathrm{~mm}$ of hydroquinone and $3 \mathrm{~mol} / \mathrm{L}$ sodium disulfite, $\mathrm{pH}$ 5.0) for 16 hours at $50^{\circ} \mathrm{C}$. Modified
TABLE 1. Characteristics of patients with ESCC

\begin{tabular}{lll}
\hline \multicolumn{1}{c}{ Characteristics } & \multicolumn{1}{c}{ Category } & No. $(\%)$ \\
\hline Sex & Male & $61(70.1)$ \\
& Female & $26(29.9)$ \\
Age (y) & $\geq 60$ & $54(62.1)$ \\
& $<60$ & $33(37.9)$ \\
Tumor differentiation & Well & $32(36.8)$ \\
& Moderate & $19(21.8)$ \\
& Poor & $36(41.4)$ \\
Depth of invasion & T1-T2 & $34(39.1)$ \\
& T3-T4 & $53(60.9)$ \\
Tumor size (cm) & $\geq 5$ & $39(44.8)$ \\
& $<5$ & $48(55.2)$ \\
Lymph node status & Negative & $52(59.8)$ \\
& Positive & $35(40.2)$ \\
Stage & Low (I and II) & $33(37.9)$ \\
& High (III and IV) & $54(62.1)$ \\
\hline
\end{tabular}

ESCC, Esophageal squamous cell carcinoma.

DNA was purified with a Qiagen gel purification kit and then treated with $0.3 \mathrm{~mol} / \mathrm{L} \mathrm{NaOH}$ for 10 minutes at room temperature. The DNA was then again purified with a Qiagen gel purification kit and eluted into $50 \mu \mathrm{L}$ of water. Methylation-specific and non-methylation-specific primers were designed by using Primer3 (Table 2), which was developed and is maintained by the Whitehead Institute/Massachusetts Institute of Technology Center of Genome Research. Complete methylation was determined based on the presence of methylation-specific and absence of non-methylation-specific PCR products. Heterozygous methylation was scored when products showed up in both methylation-specific and non-methylation-specific PCR. High annealing temperatures were used to ensure the specificity of both methylation-specific and non-methylation-specific PCR. About 20 to $50 \mathrm{ng}$ of DNA was used in each PCR amplification. After 13 minutes of heat activation, the reaction was incubated for 4 cycles of 2 minutes at $95^{\circ} \mathrm{C}, 2$ minutes at $65^{\circ} \mathrm{C}$, and then 2 minutes at $72^{\circ} \mathrm{C}$. The PCR reaction then underwent 35 to 45 cycles of 10 seconds at $95^{\circ} \mathrm{C}, 45$ seconds at $62^{\circ} \mathrm{C}$, and 30 seconds at $72^{\circ} \mathrm{C}$. A 30 -minute incubation at $72^{\circ} \mathrm{C}$ was used to finalize the PCR amplification. The products of PCR were purified by using shrimp alkaline phosphatase and exonuclease I: $1 \mathrm{U}$ of shrimp alkaline phosphatase and $6 \mathrm{U}$ of exonuclease I were added into $8 \mu \mathrm{L}$ of PCR products. The mixtures were incubated at $37^{\circ} \mathrm{C}$ for 60 minutes followed by incubation at $70^{\circ} \mathrm{C}$ for 10 minutes and sequenced (ABI3130XL).

\section{Statistical Analysis}

Statistical analysis was performed with SPSS 12.0 software. The $\chi^{2}$ test was used to compare the expression of HLA-I, LMP2, TAP1, and the promoter methylation of HLA-I between primary tumors and lymph node metastases of patients with ESCC and to assess their correlations between HLA-I antigen expression and the antigen-processing machinery (APM) component expression and the expression of HLA-I, LMP2, TAP1, and the hypermethylation of HLA promoter in ESCC lesions and clinicopathological characteristics. All statistical tests were 2-sided. Univariate analyses were conducted with the Cox proportional hazards model to assess the correlation between survival time and multiple clinicopathological variables.

\section{RESULTS}

\section{Expression of HLA-I and APM Component in Esophageal Lesions}

All the antibodies were first tested on normal esophageal tissues. As shown in Figure 1, esophageal surface epithelium 
TABLE 2. Sequencing primers used for methylation analysis

\begin{tabular}{llc}
\hline Target locus & \multicolumn{1}{c}{ Primer sequence $\left(\mathbf{5}^{\prime} \rightarrow \mathbf{3}^{\prime}\right)$} & Product size $(\mathbf{b p})$ \\
\hline HLA_AF2 & GGGTTTTTAGAGAAGTTAATTAGTGT & 257 \\
HLA_AR2 & CCAAATCTAAATCAAAACCAAAACCC & 197 \\
HLA_BF2 & GTGGGGATTTTAGAATTGGGAT & 249 \\
HLA_BR2 & CCCCACTCCCCTAAATTTCAC & 131 \\
HLA_CF2 & TGGGTGATTGGGGATTTTAGA & 255 \\
HLA_CR2 & TCCACTCCCCTAAATTTCACTTC & 151 \\
\hline
\end{tabular}

HLA, Human leukocyte antigen.

and stromal cells were homogeneously stained. Anti-HLA-I showed strong staining of cell membranes, whereas antiTAP1 and anti-LMP2 primarily stained the intracellular regions. Immunohistochemical staining of 87 primary ESCC lesions (Figure 1) was done with the aforementioned antibodies. Staining of lymphocytes was used as a reference for the immunohistochemical staining in each tissue section. Tumor lesions were found to have a highly variable expression profile ranging from a total loss to heterogeneous but decreased expression to normal expression. As shown in Table 2 and Figure 1, among the 3 proteins examined, HLA-I showed the greatest reduction in expression (ie, negative in $34.5 \%$ and downregulated in $23.0 \%$ of the lesions). TAP1 showed the smallest reduction (ie, negative in $37.9 \%$ and downregulated in $20.7 \%$ of the lesions). The expression levels of LMP2 were intermediate when compared with other proteins: LMP2 was negative in $24.1 \%$ and downregulated in $29.9 \%$ of the lesions (Table 3). Thus HLA class I antigen and APM components were all downregulated in patients with ESCC.

\section{Association of HLA Presentation With APM Components}

LMP2 participates in the proteosomal degradation of antigens. TAP1 is responsible for the transport of the antigen-derived peptide to the lumen of the endoplasmic reticulum, where they are loaded on the newly synthesized $\beta_{2}$-microglobulin-associated HLA class I heavy chains and transported through Golgi to the cell surface. ${ }^{8}$ Therefore it is conceivable that abnormal expression of any of the APM components might lead to defects in the expression of HLA-I peptide complexes. To determine whether the expression of APM components affects the presentation of HLA-I molecules, we carried out a correlation analysis. As shown in Table 4 and Figure 2, the levels of all the examined APM components were correlated to those of HLA-I molecules, suggesting that the reduced expression of HLA-I in tumor cells is partially attributed to the absence or reduction of the components involved in the steps of antigen processing and transport. In fact, a correlation between HLA-I normal osophageal tissue

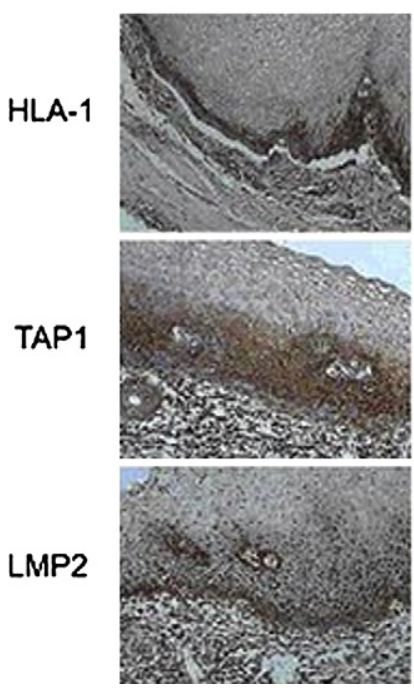

negative immunstaining in esophageal carcinoma

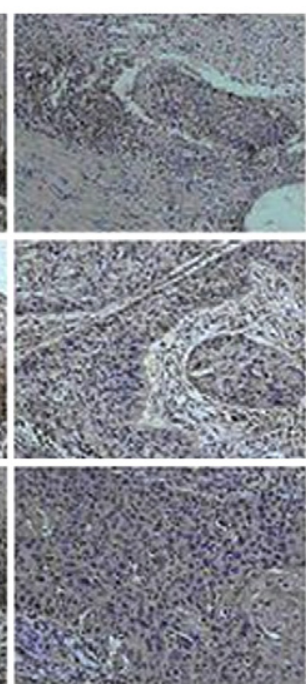

positive immunostaining in esophageal carcinoma

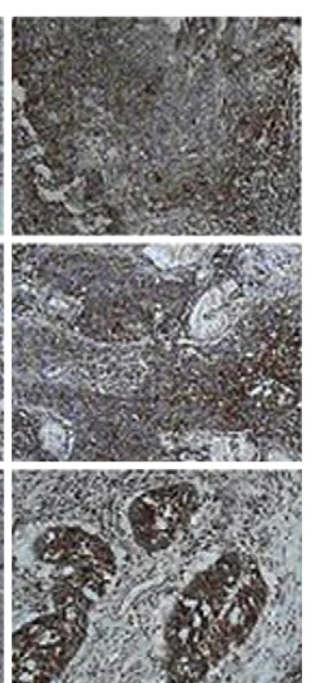

FIGURE 1. Representative staining patterns of primary esophageal squamous cell carcinoma lesions with human leukocyte antigen class I (HLA-1), transporter associated with antigen processing 1 (TAP1), and low molecular weight protein 2 (LMP2) with antigen processing-specific monoclonal antibody. Intense immunostaining for HLA-1, TAP1, and LMP2 was observed in most normal esophageal and partly esophageal carcinoma epithelia. Negative immunostaining for HLA-1, TAP1, and LMP2 was observed in most esophageal carcinomas. (Original magnification $400 \times$.) 
TABLE 3. Expression of HLA-1 and APM components in ESCC cells (positive)

\begin{tabular}{lcc}
\hline IHC parameter & $\begin{array}{c}\text { Tumor samples } \\
(\mathbf{n}=\mathbf{8 7})\end{array}$ & $\begin{array}{c}\text { Normal samples } \\
(\mathbf{n}=\mathbf{8 7})\end{array}$ \\
\hline HLA-1 & $37(42.5 \%)$ & $87(100 \%)$ \\
TAP1 & $36(41.4 \%)$ & $86(98.9 \%)$ \\
LMP2 & $40(45.9 \%)$ & $86(98.9 \%)$ \\
\hline$H L A-1$, Human leukocyte antigen class I; $A P M$, antigen-processing machinery; $E S C C$, \\
esophageal squamous cell carcinoma; IHC, immunohistochemistry; TAP1, transporter \\
associated with antigen processing $1 ; L M P 2$, low molecular weight protein 2.
\end{tabular}

expression and the levels of various APM components was previously observed in patients with laryngeal squamous cell carcinomas and ovarian cancers. ${ }^{9}$ These results suggest that pathways leading to the loss or reduction of HLA-I expression in cancer cells are probably shared among the different types of cancer.

\section{Association of HLA-I and APM Components With Clinicopathological Characteristics}

The association between the expression of HLA-I and APM components and clinicopathological characteristics was investigated in ESCC lesions. As can be seen in Table 2 , the expression levels of HLA-I molecules are significantly associated with tumor grade, the depth of tumor invasion, the status of lymph nodes, and the clinical stage but are independent of age and tumor size. TAP1 expression is significantly associated with grade, stage, and lymph node status of the tumor. LMP2 was significantly associated with tumor stage and lymph node status of the tumor. Interestingly, the expression level of LMP2 tended to be lower in patients aged 60 years or older. All the proteins examined were associated with tumor stage and lymph node status of tumor. Tumor stage and lymph node status of tumor were consistently associated with HLA-I molecules and the APM components (Table 5).

\section{Hypermethylation of $5^{\prime}$ CpG Islands in HLA-A,}

HLA-B, and HLA-C Gene Loci

The methylation status of the HLA-A, HLA-B, and HLA-C CpG islands was investigated in 87 esophageal cell carcinoma samples together with their adjacent normal epithelial tissue by using methylation-specific PCR. We detected hypermethylation of the HLA-A gene in 18 (20.6\%)

TABLE 4. Expression correlation of HLA-I with APM component $(P<.05)$

\begin{tabular}{lccccc}
\hline & \multicolumn{2}{c}{ TAP1 } & & \multicolumn{2}{c}{ LMP2 } \\
\cline { 2 - 3 } \cline { 5 - 6 } HLA-1 & Positive & Negative & & Positive & Negative \\
\hline Positive & 29 & 8 & & 23 & 14 \\
Negative & 19 & 27 & & 17 & 33 \\
\hline
\end{tabular}

$H L A-1$, Human leukocyte antigen; $A P M$, antigen-processing machinery; TAP1, transporter associated with antigen processing $1 ; L M P 2$, low molecular weight protein 2.

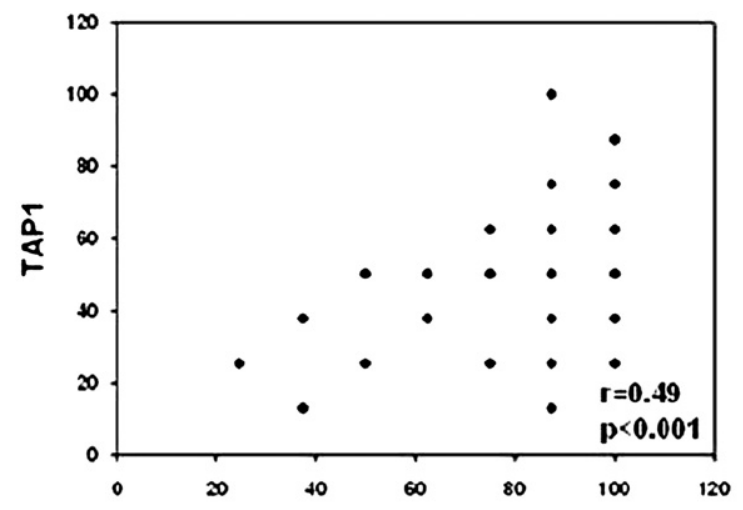

(\%)

HLA class I antigen

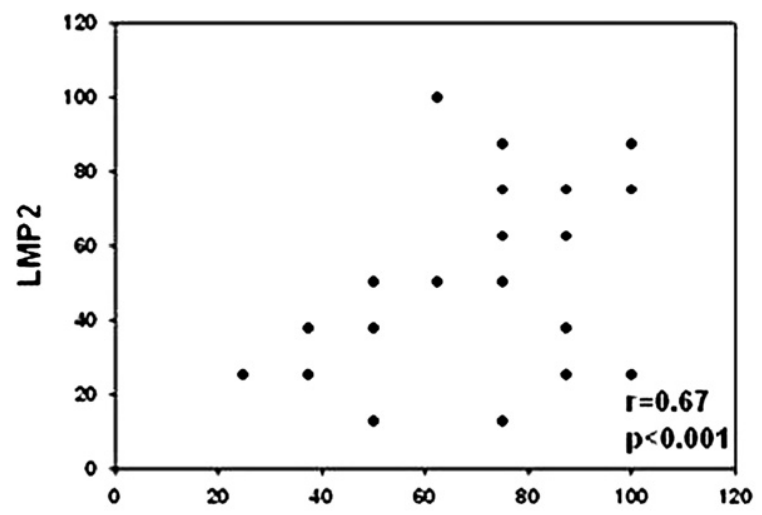

(\%)

HLA class I antigen

FIGURE 2. Correlation of human leukocyte antigen (HLA) class I expression with antigen-processing machinery component expression in primary esophageal squamous cell carcinoma lesions. The correlations between the percentages of tumor cells stained by HLA-I-specific monoclonal antibody and by transporter associated with antigen processing 1 (TAP1)specific monoclonal antibody or low molecular weight protein 2 (LMP2)specific monoclonal antibody in the lesions were analyzed with the Spearman rank correlation coefficient $(240 \times 123 \mathrm{~mm}[192 \times 192 \mathrm{DPI}])$.

cases, of HLA-B in $23(26.4 \%)$ cases, and of HLA-C in 27 $(31 \%)$ cases. These were demonstrated by the presence of products in both methylation-specific PCR and non-methylation-specific PCR (Figure 3). CpG islands in all 3 HLA class I genes were concurrently methylated in $16(18.4 \%)$ of the 87 samples. Another 15 samples harbored methylation in $\mathrm{CpG}$ islands of both the HLA-B and HLA-C genes. HLA-A alone was methylated in 9 tumor samples, HLA-B alone was methylated in 11 tumor samples, and HLA-C alone was methylated in 10 samples. No methylationspecific and only non-methylation-specific PCR signals were detected for all of the 87 normal samples. To verify the specificity of the PCR reaction, all of the products from HLA-B methylation-specific PCR were cloned and sequenced. 
TABLE 5. Correlation of HLA-I, TAP, LMP, and promoter methylation of HLA-I with clinicopathological characteristics in patients with ESCC

\begin{tabular}{|c|c|c|c|c|c|c|c|c|c|c|c|c|c|c|c|c|}
\hline \multirow[b]{2}{*}{ Characteristic } & \multicolumn{4}{|c|}{ HLA-I } & \multicolumn{4}{|c|}{ TAP1 } & \multicolumn{4}{|c|}{ LMP2 } & \multicolumn{4}{|c|}{$\begin{array}{c}\text { Promoter methylation } \\
\text { of HLA }\end{array}$} \\
\hline & + & \pm & - & $P$ value & + & \pm & - & $P$ value & + & \pm & - & $P$ value & $H^{*}$ & $+^{*}$ & - & $P$ value \\
\hline \multicolumn{17}{|l|}{ Sex } \\
\hline Male & 26 & 14 & 21 & .908 & 23 & 12 & 26 & .725 & 31 & 16 & 14 & .203 & 22 & 20 & 19 & .425 \\
\hline Female & 11 & 6 & 9 & & 13 & 6 & 7 & & 9 & 10 & 7 & & 9 & 10 & 7 & \\
\hline \multicolumn{17}{|l|}{ Age (y) } \\
\hline$\geq 60$ & 24 & 10 & 20 & .001 & 21 & 10 & 23 & .424 & 29 & 16 & 9 & .174 & 17 & 18 & 20 & .071 \\
\hline$<60$ & 13 & 10 & 10 & & 15 & 8 & 10 & & 11 & 10 & 12 & & 14 & 12 & 6 & \\
\hline \multicolumn{17}{|c|}{ Tumor differentiation } \\
\hline Well & 13 & 10 & 9 & .05 & 15 & 8 & 9 & .306 & 13 & 11 & 8 & .05 & 10 & 13 & 9 & .05 \\
\hline Moderate & 9 & 4 & 6 & & 7 & 2 & 10 & & 8 & 6 & 5 & & 7 & 6 & 6 & \\
\hline Poor & 15 & 6 & 15 & & 14 & 8 & 14 & & 19 & 9 & 8 & & 14 & 11 & 11 & \\
\hline \multicolumn{17}{|l|}{ Depth of invasion } \\
\hline $\mathrm{T} 1-\mathrm{T} 2$ & 20 & 9 & 5 & .047 & 16 & 6 & 12 & .818 & 15 & 11 & 8 & .206 & 11 & 5 & 18 & .007 \\
\hline $\mathrm{T} 3-\mathrm{T} 4$ & 17 & 11 & 25 & & 20 & 12 & 21 & & 25 & 15 & 13 & & 20 & 25 & 8 & \\
\hline \multicolumn{17}{|l|}{ Tumor size } \\
\hline$\geq 5 \mathrm{~cm}$ & 18 & 6 & 15 & .284 & 12 & 5 & 20 & .096 & 18 & 9 & 12 & .196 & 21 & 17 & 6 & .021 \\
\hline$<5 \mathrm{~cm}$ & 19 & 14 & 15 & & 24 & 13 & 13 & & 22 & 17 & 9 & & 10 & 13 & 25 & \\
\hline \multicolumn{17}{|l|}{ Lymph node status } \\
\hline Positive & 15 & 6 & 14 & .002 & 11 & 8 & 16 & .188 & 17 & 10 & 8 & .013 & 17 & 13 & 5 & .002 \\
\hline Negative & 22 & 14 & 16 & & 25 & 10 & 17 & & 23 & 16 & 13 & & 14 & 17 & 21 & \\
\hline \multicolumn{17}{|l|}{ Stage } \\
\hline I-II & 17 & 10 & 6 & .029 & 18 & 7 & 8 & .282 & 17 & 9 & 8 & .034 & 12 & 9 & 12 & .011 \\
\hline III-IV & 20 & 10 & 24 & & 18 & 11 & 25 & & 23 & 17 & 13 & & 19 & 21 & 14 & \\
\hline
\end{tabular}

HLA-I, Human leukocyte antigen class I; TAP, transporter associated with antigen processing; $L M P$, low molecular weight protein; ESCC, esophageal squamous cell carcinoma; + , positive; \pm , downregulation; -, negative; +**, HLA-A, HLA-B, HLA-C, or 2 of them; +*, HLA-A, HLA-B, or HLA-C.

\section{Association of Hypermethylation of Promoter of \\ HLA-I With HLA Presentation and \\ Clinicopathological Characteristics}

The association between the hypermethylation of the promoter of HLA-I and clinicopathological characteristics was investigated in ESCC lesions. As can be seen in Table 4, the methylated levels of $5^{\prime} \mathrm{CpG}$ islands in HLA are significantly associated with tumor grade, the depth of tumor invasion, the status of lymph nodes, and the clinical stage but are independent of age and tumor size, as shown in the HLA-I and APM component expression (Table 5). Also, to further assess whether the hypermethylation of HLA-I promoter in the primary tumor represented a prognostic parameter in patients with ESCC, we used the Cox proportional hazards model and made the survival curve (Figure 4). The covariate parameters included several clinicopathological factors in addition to DNA hypermethylation. On univariate analysis, several factors, including hypermethylation of HLA-I, showed a significantly higher hazard ratio for a poor prognosis. Those indicated that the DNA of HLA-I

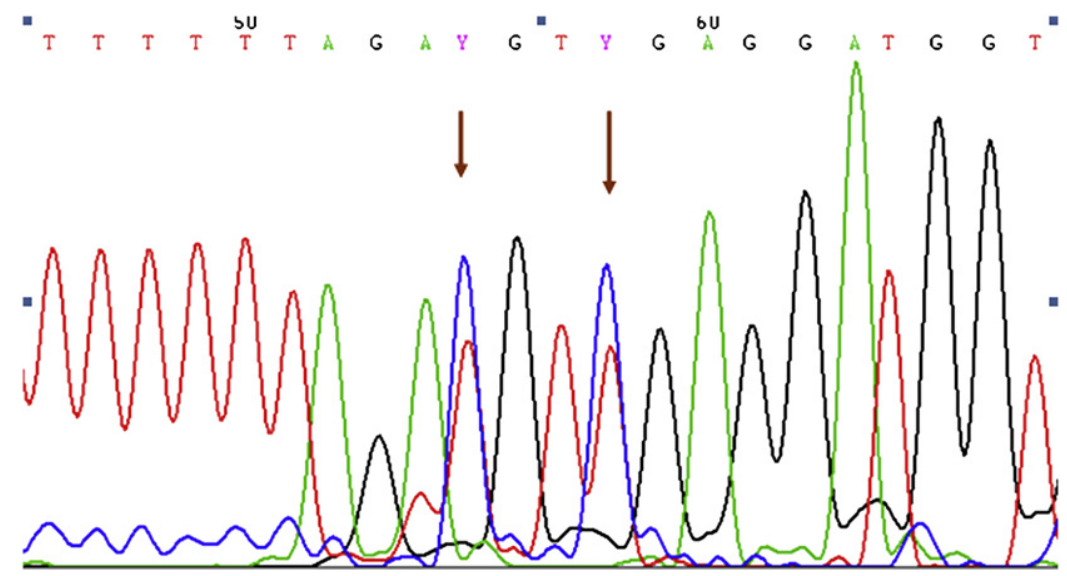

FIGURE 3. Methylation analysis of the promoter of the human leukocyte antigen class I gene by sequencing. The arrows depict the methylated CpG site (Y representing the T/C SNP). The different-colored curve represent the 4 bases: red, T; green, A; black, G; blue, Y/C. 


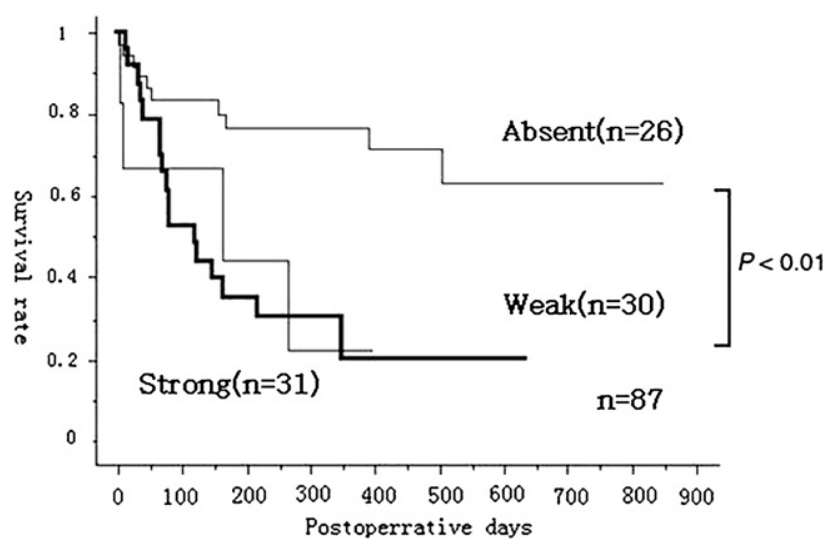

FIGURE 4. Survival curve in patients with esophageal squamous cell carcinoma. The median survival time in strong expression was significantly shorter than in absent expression in terms of DNA hypermethylation.

hypermethylation in patients with ESCC was closely related to a poor prognosis (Table 6).

\section{DISCUSSION}

The HLA-I antigens HLA-A, HLA-B, and HLA-C, which form the class I major histocompatibility complex in human subjects, take part in the recognition of virally infected, grafted, or transformed cells by cytotoxic $\mathrm{T}$ cells. ${ }^{3}$ The HLA genes are located on chromosome 6p21 and are expressed in most somatic tissues. Selective loss of expression of these loci has frequently been observed in human tumors, and this event is thought to help tumor cells escape immune surveillance by $\mathrm{T}$ cells and natural killer cells. ${ }^{10,11}$ The downregulation of HLA-I has been observed in kidney, prostate, stomach, colon, and germ cell testicular cancers and has been associated with tumor invasiveness and aggressiveness. ${ }^{10}$ In our study we found that HLA-I was downregulated or even lost in $78.2 \%$ of primary ESCC lesions and was downregulated in $87 \%$ of metastatic lymph nodes. This frequency in primary ESCC lesions is comparable with frequencies reported in previous studies. ${ }^{12,13}$ Those studies and our results clearly show that there is a marked reduction in the expression of HLA-I molecules in ESCC lesions, and in our studies we showed that the reduction of HLA-I presentation was significantly associated with metastasis, tumor grade, and poor survival in patients with ESCC. This is also comparable with results reported in previous studies. ${ }^{14}$
Loss of HLA-I antigen expression can occur at the genetic, transcriptional, and posttranscriptional levels. DNA methylation, especially $5^{\prime} \mathrm{CpG}$ methylation, is an important mechanism in silencing the expression of genes. ${ }^{15,16}$ DNA methylation can directly interfere with the basal transcriptional machinery by altering the DNA secondary structure, especially the major groove conformation. DNA methylation can also induce chromosomal remodeling through histone deacetylation, resulting in transcriptional repression. A group of methyl-CpG-binding proteins, which preferentially bind to methylated $\mathrm{CpG}$ dinucleotides, might interact with $\sin 3 \mathrm{~A}$. Sin $3 \mathrm{~A}$ in turn interacts with histone deacetylase ${ }^{17}$ and is thought to be involved in this process. $^{18,19}$ Abnormal DNA methylation has been found in various genes, including the putative tumor suppressor genes retinoblastoma and p16INK4A, leading to their downregulation in tumors. ${ }^{20}$ Costello and colleagues ${ }^{21}$ conducted a global examination of $\mathrm{CpG}$ methylation in a large group of tumors and observed a tumor type-specific pattern. $\mathrm{CpG}$ islands are small regions of DNA ranging from 0.5 to 4 or $5 \mathrm{~kb}$ in length where the GC content is greater than $60 \%$ and the $\mathrm{CpG} / \mathrm{GpC}$ ratio is greater than $0.6 .{ }^{16}$ Using these criteria, we have conducted a search for well-preserved $\mathrm{CpG}$ islands in genes that have been reported to be inactivated by high $\mathrm{CpG} / \mathrm{GpC}$ ratios, as well as a GC content of greater than $60 \%$. Hypermethylation of these genes has not been reported in either tumor or spermatogenic cells in which HLA-I genes were not expressed. ${ }^{22}$

$\mathrm{CpG}$ island hypermethylation has been suggested to be one of the major mechanisms for inactivation of genes that inhibit tumor progression and infiltration. ${ }^{16}$ Each of the 3 HLA-I genes contains a well-preserved $\mathrm{CpG}$ island in its $5^{\prime}$ region. Methylation-specific primers were designed to amplify the region within or close to the promoters where methylation has the greatest effect on transcription. Hypermethylation of each of the 3 loci was observed in some patients with ESCC, and methylation of HLA-B was confirmed by means of sequencing. The HLA-B locus was the most frequently hypermethylated, which is consistent with the observation that in human tumors downregulation of HLA class I expression was B locus specific. ${ }^{23}$ In this study we analyzed the association between the expression of HLA-I with the hypermethylation of its promoters and concluded that they have an inverse correlation. In

TABLE 6. Univariate analysis of patients

\begin{tabular}{llcccc}
\hline & & \multicolumn{2}{c}{ Univariate analysis, overall survival } & & \multicolumn{2}{c}{ Multivariate analysis, overall survival } \\
\multicolumn{1}{c}{ Variables } & \multicolumn{1}{c}{ Categories } & HR $(\mathbf{9 5} \% \mathbf{C I})$ & $\boldsymbol{P}$ value & HR (95\% CI) & \multicolumn{1}{c}{$\boldsymbol{P}$ value } \\
\hline Sex & Male (vs female) & $1.75(0.85-3.59)$ & .13 & \\
Tumor size $(\mathrm{cm})$ & $\geq 5($ vs $<5)$ & $2.43(1.18-5.21)$ & .02 & $1.79(0.71-4.53)$ \\
Hypermethylation & Positive (vs negative) & $4.03(1.87-8.69)$ & $<.01$ & $3.41(1.42-8.33)$ \\
Tumor differentiation & Poorly (vs well and moderate) & $2.74(1.31-5.75)$ & $<.01$ & .19 \\
\hline
\end{tabular}

$H R$, Hazard ratio; 95\% CI, 95\% confidence interval. 
esophageal epithelial tissue about $67 \%$ of promoters of HLA-I have been detected methylated; meanwhile, the expression of HLA-I antigen was downregulated synchronously. Furthermore, the methylation of HLA-I increases with disease progression, with markedly less frequency in primary lesions than in lymph node metastasis, and it was significantly associated with tumor grade. Therefore we conclude that DNA hypermethylation is a mechanism for loss of expression of the HLA-I genes in human ESCC. Activation of HLA-I antigen expression has been proposed as a therapeutic approach for ESCC. ${ }^{24}$ Reactivation of tumor suppressor genes through inhibition of DNA methylation has been tested so as to inhibit cancer development. ${ }^{25}$ In fact, it has been reported that the demethylating agents 5 -azacytidine and 5-aza-2'-deoxycytidine can restore the normal demethylated state of several types of tumor suppressor genes and increase their expression in various cancers, including esophageal cancer, in vitro and in vivo. ${ }^{26-28}$ Methylation of genes in tumor cells could provide a tumor-specific target for new therapies. ${ }^{29,30}$ However, to date, a clinical trial on demethylating agents in esophageal cancer is unavailable. Although it is too early to have any expectation about the effect of these drugs on esophageal cancer, this is a very promising concept and needs to be tested in clinical trials. These approaches need to be studied further.

\section{References}

1. Goldminc M, Maddern G, Le Prise E, Meunier B, Campion JP, Launois B. Oesophagectomy by a transhiatal approach or thoracotomy: a prospective randomized trial. Br J Surg. 1993;80:367-70.

2. Dunn GP, Bruce AT, Ikeda H, Old LJ, Schreiber RD. Cancer immunoediting: from immunosurveillance to tumor escape. Nat Immunol. 2002;3:702-9.

3. Mizuki N, Ando H, Kimura M, Ohna S, Miyata S, Yamazaki M, et al. Nucleotide sequence analysis of the HLA class I region spanning the 273-kb segment around the HLA-B and-C genes. Genomics. 1997;42:55-66.

4. Zingg JM, Jones PA. Genetic and epigenetic aspects of DNA methylation on genome expression, evolution, mutation and carcinogenesis. Carcinogenesis. 1997; 18:869-82.

5. Baylin SB, Herman JG, Graff JR, Vertino PM, Issa JP. Alterations in DNA methylation: a fundamental aspect of neoplasia. Adv Cancer Res. 1998;72:141-96.

6. Garrido F, Cabrera T, Accolla RS, et al. HLA and cancer: 12th International Histocompatibility Workshop Study. In: Charron D, ed. HLA genetic diversity of HLA. Vol 1. Functional and medical implication. Paris: EDK; 1997:445-52.

7. Herman JG, Graff JR, Myohanen S, Nelkin BD, Baylin SB. Methylation-specific PCR: a novel PCR assay for methylation status of CpG islands. Proc Natl Acad Sci U S A. 1996;93:9821-6.

8. Flutter B, Gao B. MHC class I antigen presentation: recently trimmed and well presented. Cell Mol Immunol. 2004;1:22-30.
9. Ogino T, Shigyo H, Ishii H, Katayama A, Miyokawa N, Harabuchi Y, et al. HLA class I antigen down-regulation in primary laryngeal squamous cell carcinoma lesions as a poor prognostic marker. Cancer Res. 2006;66:9281-9.

10. Rui-Cabello F, Klein E, Garrido F. MHC antigen on human tumors. Immunol Lett. 1991;29:181-9.

11. Browning M, Dunnion D. HLA and cancer: implications for cancer immunotherapy and vaccination. Eur J Immunogenet. 1997;24:293-312.

12. Nie Y, Yang G, Song Y, Zhao X, So C, Liao J, et al. DNA hypermethylation is a mechanism for loss of expression of the HLA class I genes in human esophageal squamous cell carcinomas. Carcinogenesis. 2001;22:1615-23.

13. Hosch SB, Izbicki JR, Pichlmeier U, Stoecklein N, Niendorf A, Knoefel WT, et al. Expression and prognostic significance of immunoregulatory molecules in esophageal cancer. Int J Cancer. 1997;74:582-7.

14. Mizukami Y, Kono K, Maruyama T, Watanabe M, Kawaguchi Y, Kamimura K, et al. Downregulation of HLA class I molecules in the tumor is associated with a poor prognosis in patients with esophageal squamous cell carcinoma. Br J Cancer. 2008;99:1462-7.

15. Zingg JM, Jones PA. Genetic and epigenetic aspects of DNA methylation on genome expression, evolution, mutation and carcinogenesis. Carcinogenesis. 1997; 18:869-82.

16. Baylin SB, Herman JG, Graff JR, Vertino PM, Issa JP. Alterations in DNA methylation: a fundamental aspect of neoplasia. Adv Cancer Res. 1998;72:141-96.

17. Nan X, Ng HH, Johnson CA, Laherty CD, Turner BM, Eisenman RN, et al. Transcriptional repression by the methyl-CpG-binding protein MeCP2 involves a histone deacetylase complex. Nature. 1998;393:386-9.

18. Bird AP, Wolffe AP. Methylation-induced repression-belts, braces and chromatin. Cell. 1999;99:451-4.

19. Hendrich B, Bird A. Mammalian methyltransferases and methyl-CpG- binding domains: protein involved in DNA methylation. Curr Top Microbiol Immunol. 2000;294:55-74.

20. Jones PA, Laird PW. Cancer epigenetics comes of age. Nat Genet. 1999;21:163-7.

21. Costello JF, Fruhwald MC, Smiraglia DJ, Rush LJ, Robertson GP, Gao X, et al. Aberrant $\mathrm{CpG}$ - island methylation has non-random and tumor-type-specific patterns. Nat Genet. 2000;24:132-8.

22. Guillaudeux T, Gomez E, Onno M, et al. Expression of HLA class I genes in meiotic and post-meiotic human spermatogenic cell. Biol Reprod. 1996;55: 99-110.

23. Griffioen M, Ouwerkerk IJ, Harten V, Schrier PI. HLA-B down-regulation in human melanoma is mediated by sequences located downstream of the transcriptioninitiation site. Cancer. 1999;80:573-80.

24. Debruyne L. Treatment of malignancy by direct gene transfer of a foreign MHC class I molecule. Cancer Immunol Immunother. 1996;43:180-9.

25. Momparler RL, Eliopoulos N, Ayoub J. Evaluation of an inhibitor of DNA methylation,5-aza-2'-deoxycytidine, for the treatment of lung cancer and the future role of gene therapy. Adv Exp Med Biol. 2000;465:433-46.

26. Esteller M. Relevance of DNA methylation in the management of cancer. Lancet Oncol. 2003;4:4351-8.

27. Ehrlich M. DNA methylation in cancer:too much, but also too little. Oncogene. 2002;21:5400-13

28. Esteller M. CpG island hypermethylation and tumor suppressor genes:a booming present, a brighter future. Oncogene. 2002;21:5427-40.

29. van Groeningen CJ, Leyva A, O'Brien AM, Gall HE, Pinedo HM. Phase I and pharmacokinetic study of 5-aza-2 deoxycytidine(NSC 127716)in cancer patients. Cancer Res. 1986;46:4831-6.

30. Aparicio A, Eads CA, Leong LA, Laird PW, Newman EM, Synold TW, et al. Phase I trial of continuous infusion 5-aza-2-deoxvcvtidine. Cancer Chemother Pharmacol. 2003;51. 231-23. 